\title{
Características germinativas de dos especies vegetales promisorias para el mejoramiento genético de
} especies comerciales

\section{Germinating characteristics of two promising plant species for genetic improvement of comercial species}

Freddy Mejía Coico ${ }^{1}$, José Mostacero León ${ }^{1 *}$, Jordan de la Cruz Castillo ${ }^{1}$ y Danilo Gastañadui Rosas ${ }^{1}$

\section{RESUMEN}

Las actividades antrópicas negativas y crecientes, han provocado la inestabilidad de muchos ecosistemas en el planeta. Es así que las lomas, ecosistemas sui géneris de las costas del Perú y Chile, se han constituido en ecosistemas muy frágiles y por tanto de atención prioritaria de la investigación para lograr su conservación como fuente de recursos que su biodiversidad puede brindar al hombre. Puesto que estos ecosistemas convenientemente administrados y gestionados pueden constituirse en reales fuentes de desarrollo y progreso, este trabajo determinó las características germinativas de Solanum pimpinellifolium y S. habrochaites como especies promisorias para el mejoramiento genético de otras variedades de Solanum lycopersicum (tomates comerciales).

El trabajo se inició con la colecta de estas especies, cuya determinación taxonómica fue confirmada en el Herbarium Truxillense (HUT), para luego trasladarlas al laboratorio de la UNT donde se siguieron todas las etapas para determinar las características germinativas. Se concluye que: Porcentaje de germinación, Velocidad de germinación, Uniformidad de germinación, Porcentaje de emergencia y Valor de la germinación para Solanum pimpinellifolium son: $76 \%, 4,87$ días, 6,33 días, $55,33 \%$ y 31,02, respectivamente, meinetras que para Solanum habrochaites son: $83 \%, 5.6$ días, 6,67 días, 49,33\% y 116,17 , respectivamente.

Palabras clave: Características germinativas, Solanum pimpinellifolium, S. habrochaites, Cerro Campana.

\begin{abstract}
Negative and increasing anthropogenic activities have led to the instability of many ecosystems on the planet. It is thus that the hills, sui generis ecosystems of the coasts of Peru and Chile, have become very fragile ecosystems and therefore of priority attention of the investigation to obtain its conservation as a source of resources that its biodiversity can offer to the man. Since these properly managed and managed ecosystems can be real sources of development and progress, this work determined the germinative characteristics of Solanum pimpinellifolium and $S$. habrochaites as promising species for the genetic improvement of other varieties of Solanum lycopersicum (comercial tomatoes).

The work began with the collection of these species, whose taxonomic determination was confirmed in the Herbarium Truxillense (HUT), and then transferred to the laboratory of the UNT where all steps were followed to determine the germinative characteristics. It is concluded that: Percentage of germination, Germination rate, Germination uniformity, Emergency percentage and Germination value for Solanum pimpinellifolium are: 76\%, 4.87 days, 6.33 days, $55.33 \%$ and 31.02 , respectively, that for Solanum habrochaites are: 83\%, 5.6 days, 6.67 days, $49.33 \%$ and 116.17 , respectively.
\end{abstract}

Keywords: Germination characteristics, Solanum pimpinellifolium, S. habrochaites, Cerro Campana.

\footnotetext{
Universidad Nacional de Trujillo, Trujillo, La Libertad, Perú

"Autor de correspondencia. E-mail:jmostacero@unitru.edu.pe
} 


\section{INTRODUCCIÓN}

Las lomas, llamadas alguna vez "oasis de neblina", son formaciones geográficas que se encuentran ubicadas a lo largo de la árida franja costera de América del Sur desde los $8^{\circ}$ L.S en Trujillo, hasta el norte de Chile $\left(30^{\circ}\right.$ L.S), entre Huasco y Coquimbo (Ferreyra, 1979; Mostacero, 1987; Mostacero et al., 2007; Quiroz, 2008).

Estas lomas conforman comunidades vegetales, que son asociaciones únicas en el mundo y constituidas preponderantemente por hierbas anuales y perennes (tuberíferas, rizomatosas y bulbígenas), distribuidas en una gran diversidad biológica de familias, géneros $\mathrm{y}$ especies. Muchas de estas son endémicas, y a las que se asocian muchas especies de insectos, caracoles, arañas, serpientes, aves, vizcachas, y otros mamíferos. Estas comunidades bióticas, se deben al fenómeno de inversión térmica, que produce el enfriamiento de las nubes procedentes del mar y que luego se condensan en agua líquida de "garúas" y "lloviznas", haciendo posible la cobertura vegetal anual de estas lomas (Canziani, 1998; Carranza, 1996; Mostacero et al., 2007; Lamb, 2011).

Las lomas del Cerro Campana y Cabezón son importantes no sólo por ser el límite norte de las Comunidades Lomales, sino por la composición florística y faunística variada y de mucho valor para la alimentación, medicina, forraje, vestido, así como por ser fuente de germoplasma para la obtención de nuevas variedades comerciales de tomates, caiguas, papas, quinuas, etc., de menor ciclo vegetativo, de mayor calidad y cantidad de elementos nutritivos y de principios activos, resistentes a plagas y sequías y para combatir la desertificación.

Estas comunidades bióticas poseen especies silvestres de los géneros Solanum (papas y tomates), Cyclanthera (caigua), o Chenopodium (quinua). De lo mencionado en esta investigación se ha dirigido la atención a especies silvestres de Solanum (tomates silvestres), para luego continuar con otras especies y mejorar así especies cultivadas de estos ejemplares, para así obtener ventajas prioritarias, ya que estas comunidades, día a día, vienen siendo amenazadas por una serie de actividades antrópicas negativas y de no ser propagadas y/o conservadas, tanto in situ como ex situ, se extinguirían y pondrían en riesgo la integridad de este ecosistema (Mostacero, 1987; Sagástegui et al., 1988).

En tal sentido, es importante centralizar esfuerzos para conservar estas extraordinarias especies, y que en un futuro, no muy lejano se constituyan en verdaderos recursos para el desarrollo sostenible y sustentable del país, pues el enorme potencial tanto agrícola como medicinal y ornamental que poseen amerita ejercer múltiples esfuerzos para su conservación. Las “especies vegetales lomales" hasta hoy, es poca la atención que han recibido, y según la bibliografía es poco lo que se conoce. Por estas razones se realiza esta investigación, con énfasis en su fenología y sus características germinativas, que abran las puertas a investigaciones genéticas posteriores encaminadas al bienestar y economía del hombre (Mostacero et al., 2007).

\section{Historial de especies}

Iniciando con Solanum pimpinellifolium L., "tomatillo silvestre", es especie herbácea muy común en las costas de Perú y Ecuador; rara vez es cosechada para consumo humano, y ampliamente usada como fuente de germoplasma por los mejoradores de tomate al ser fácilmente hibridizada con S. lycopersicum L. (Harman y Bale, 1994; Bai y Lindhout, 2007; Zuriaga et al., 2009).

De igual manera Solanum habrochaites S. Knapp \& D.M. Spooner, “tomatillo", son arbustos decumbentes o enredaderas perennes de hasta seis metros de longitud. De olor peculiar y desagradable para el hombre, sin embargo, pueden ser características muy importante si se tienen en cuenta como "plantas trampa" o insecticidas para ahuyentar o matar muchas plagas (Rick, 1991). Sus frutos, de 1 a 1,5 cm de diámetro, globosos, biloculares y verde pálido a intenso. Las semillas son ovaladas, de 2 a $3 \mathrm{~mm}$ de longitud y de color marrón oscuro; pueden ser utilizadas como fuente de germoplasma para la obtención de variedades precoces, de resistencia a la sequedad y de alta calidad 
y cantidad de frutos al ser cruzados con especies de tomates cultivados (Rick et al., 1979).

Conocer aspectos básicos de estas especies como fenología y características germinativas permiten tener las bases para la mejora genética, y obtener variedades resistentes a plagas potenciales en el cultivo del tomate como son: Tuta absoluta (Leite et al., 2001), Tetranichus urticae (Guo et al., 1993), Spodoptera exigua (Lin et al., 1987), Heliothis Zea (Dimock, 1983), Plusia chalcites, Heliothis armigera, Trialeurodes vaporarium (Juvik, 1982), Aphis spp. (Williams et al., 1980) Myzus persicae (Kenedy y Yamamoto, 1979), y Lyriomiza spp. (Webb et al., 1971). Esto debido a la secreción por los tricomas de determinados compuestos químicos (Kalloo, 1993).

Así mismo, presenta resistencias a diversas enfermedades causadas por hongos como Phytophthora infestans y Alternaria solani, a bacteriosis como Corynebacterium michiganense y Pseudomonas tomato, a diferentes virosis como TSWV, TMV, TLCV, TYLCV, y a los nematodos Globodera pallida y diferentes especies del género Meloidogyne (Cortada et al., 2009).

Estas especies silvestres de tomates son tolerantes al frío y a las heladas (Paterson et al., 1987), y presentan caracteres de interés en la mejora de la calidad, como un elevado contenido en azúcares y ácidos orgánicos (Bucheli et al., 1999, Roselló y Nuez, 2006), por lo que es indispensable conocer las características germinativas de esta especie, al no existir registro alguno sobre ellas.

Por lo descrito anteriormente se planteó determinar las características germinativas de Solanum pimpinellifolium y S. habrochaites; especies promisorias para el mejoramiento genético de muchas especies comerciales, como una forma de sentar las bases para futuros proyectos tanto biotecnológicos como de conservación y aprovechamiento de nuestros recursos vegetales, al no existir bases bibliográficas acerca de la fenología y más exactamente acerca de sus características germinativas, que abran las puertas a investigaciones posteriores encaminadas a la conservación de nuestros recursos.

\section{MATERIAL Y MÉTODOS}

El material biológico requerido para esta investigación lo constituyeron los frutos de Solanum pimpinellifolium y S. habrochaites, que fueron colectados en las comunidades bióticas del "Cerro Campana", ubicado a la altura del Km. 576 de la Panamericana Norte, Huanchaco, Trujillo (Departamento de La LibertadPerú), entre los $7^{\circ} 58^{\prime} 54.62^{\prime \prime} L S$ y $79^{\circ} 06^{\prime} 18.04$ 'LO (Figura 1).

Entre abril y octubre de 2015 se colectaron plantas completas y/o parte de ellas (tallos, hojas, flores y frutos), para luego ser colocadas en las prensas botánicas y cumplir con el proceso de herborización correspondiente. También se colectaron frutos maduros de las dos especies, que debidamente etiquetados fueron colocados en bolsas de papel para trasladarlos al laboratorio para su posterior tratamiento germinativo.

El material herborizado fue trasladado al Herbarium Truxillense (HUT) de la Universidad Nacional de Trujillo (UNT), para realizar la determinación taxonómica por comparación con el material registrado en el herbario, mediante el "corrido de claves taxonómicas" o para enviar algunas muestras a especialistas extranjeros del Missouri Botanical Garden para su verificación.

Las muestras de los frutos se llevaron al laboratorio de botánica, en el Departamento de Ciencias Biológicas del Pabellón Antonio Samanamud Romero de la Universidad Nacional de Trujillo. Allí, se extendieron los frutos maduros en papel Bond de 80 gr, y luego se pesaron y midieron. Seguidamente se cortaron y se extrajeron las semillas, que fueron contadas y pesadas, y luego seleccionadas y almacenadas en recipientes de vidrio debidamente etiquetados para su posterior procesamiento.

El proceso de germinación para determinar las características germinativas de las semillas de estas tres especies se realizó de la siguiente manera: Para evaluar el "Porcentaje de germinación" de Solanum pimpinellifolium y $S$. habrochaites, por 
fórmula:

Valor de germinación $=$ VGD final $x$ Valor máx. VGD

Dónde:

VGD final= Es el porcentaje final de la germinación dividido entre el número de días de posible duración de la prueba.

Valor máx. VGD= Es el valor más alto del porcentaje de germinación dividido entre el número de días que se requirió para llegar a ese punto.

Para determinar el "Porcentaje de emergencia" de Solanum pimpinellifolium y S. habrochaites, se esterilizaron tres bandejas de tecnopor de 20,5 cm de largo, $14 \mathrm{~cm}$ de ancho y $2,5 \mathrm{~cm}$ de altura, con algodón humedecido en una solución de lejía al $2 \%$ durante diez minutos, utilizando como sustrato una mezcla de arena, musgo y humus en proporción 1:1:1, previamente esterilizados. Posteriormente se seleccionaron 150 semillas por cada una de las dos especies y se sembraron en tres repeticiones de 50 semillas por bandeja. Las bandejas de tecnopor fueron selladas con plástico adherente debidamente rotuladas. Cabe mencionar que se les brindó las condiciones óptimas de temperatura, $22 \pm 2^{\circ} \mathrm{C}$, para favorecer la germinación. El riego se le aplicó cada tres días o según los requerimientos de la futura planta. Se consideró plántula emergida aquella cuya parte superior tomó la forma de un bastón curvado.

\section{RESULTADOS}

Las características germinativas de Solanum pimpinellifolium y $S$. habrochaites, comúnmente llamados tomates silvestres, procedentes de la Loma "Cerro Campana", se presentan analíticamente en la tabla 1. De su análisis se establece que, en condiciones de laboratorio, estas especies presentan claramente un alto porcentaje de germinación, $76 \%$ y $83 \%$, respectivamente. También se muestran los valores para la "Velocidad de germinación", la "Uniformidad de germinación", el "Porcentaje de emergencia", y el "Valor de la germinación"; estos resultados fueron los siguientes, en el orden antes descrito: 4,87 días, 6,33 días, 55,33\% y 31,02 para Solanum pimpinellifolium, y de 5,6 días, 6,67 días, 49,33\%, 116,17 para Solanum habrochaites. Estos valores corroboran el alto grado de adaptabilidad a las cambiantes condiciones ambientales reinantes en las lomas, probablemente como consecuencia de los genes conseguidos por evolución tras miles de años y que pueden utilizarse ventajosamente para el mejoramiento de especies de “tomates comerciales" (Solanum lycopersicum).

Tabla 1. Características Germinativas de Solanum pimpinellifolium y S. habrochaites "tomates silvestres" de la loma "Cerro Campana", La Libertad, Perú

\section{Características Germinativas}

\section{Especies}

\section{Solanum pimpinellifolium Solanum habrochaites}

\begin{tabular}{ccc}
\hline Porcentaje de germinación & $76 \%$ & $83.00 \%$ \\
Velocidad de germinación & 4.87 días & 5.6 días \\
Uniformidad de germinación & 6.33 días & 6.67 días \\
Porcentaje de emergencia & $55.33 \%$ & $49.33 \%$ \\
Valor de la germinación & 31.02 & 116.17 \\
\hline
\end{tabular}




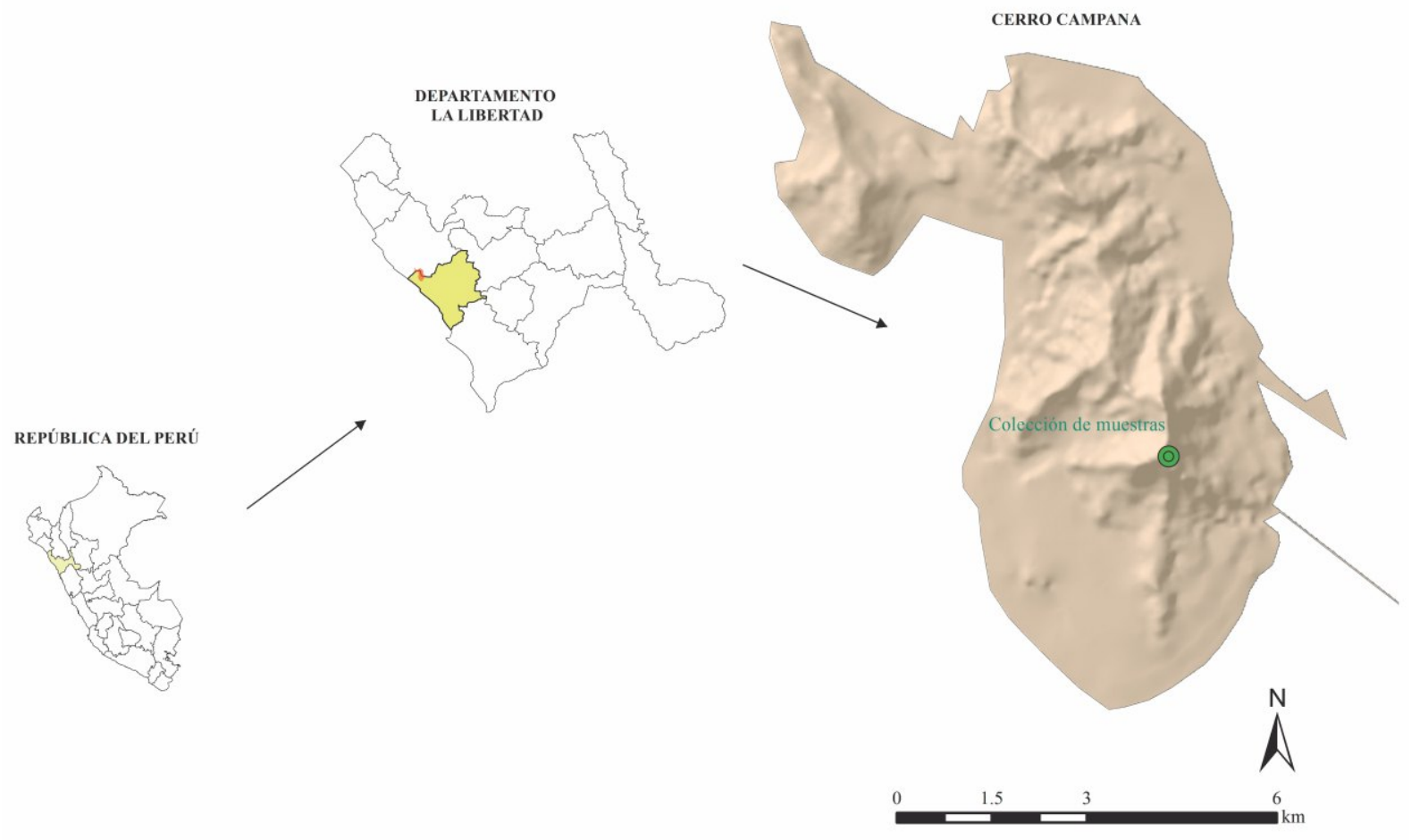

Figura 1. Mapa de ubicación geográfica del área de estudio.

especie, se esterilizaron tres bandejas de tecnopor de $20,5 \mathrm{~cm}$ de largo, $14 \mathrm{~cm}$ de ancho y 2,5 $\mathrm{cm}$ de altura, previamente lavadas y desinfestadas con una solución de lejía al $2 \%$ durante diez minutos. Posteriormente, en cada bandeja se colocaron ocho discos de papel secante estériles y humedecidos con agua destilada. A continuación se procedió a uniformizar 150 semillas por cada una de las dos especies, que se distribuyeron en tres grupos de 50 semillas cada una. Estas, debidamente rotuladas y selladas con plástico adherente, fueron puestas en condiciones de $22 \pm 2^{\circ} \mathrm{C}$ para favorecer la germinación. Se aplicó riego cada tres días, o según los requerimientos de la futura planta. Diariamente, las bandejas fueron examinadas para cuantificar las semillas que iban germinando, considerándose semilla germinada aquella cuya radícula medía $0,5 \mathrm{~cm}$ de longitud o más. Para la toma de datos se tuvo muy en cuenta la hora del día. El "Porcentaje de germinación" se determinó a través de la relación entre el total de semillas sembradas y el total de semillas germinadas:

\section{$\%$ de Germinación $=$ TG $/ T S \times 100$}

Dónde:

$T G=$ Total de semillas germinadas

$T S=$ Total de semillas sembradas

Para determinar la "Velocidad de germinación" de Solanum pimpinellifolium y $S$. habrochaites, se utilizaron los cuartiles germinativos (D25, D50 y D75), de acuerdo con la metodología propuesta por Morales y Camacho (1985).

Para determinar la "Uniformidad de germinación" de Solanum pimpinellifolium y $S$. habrochaites, se determinó contabilizando el número de días que tardaron las semillas en alcanzar entre el 5\% y la germinación final, para cada unidad experimental de esta especie.

Para determinar el "Valor de la germinación" de Solanum pimpinellifolium y $S$. habrochaites, se calculó a través del valor compuesto de Czabator, para ello se utilizaron los datos obtenidos en la prueba de "Porcentaje de germinación"; a través de la siguiente 
Reforzando estos resultados, las figuras 2 y 3 , muestran claramente el total acumulado en días de semillas germinadas tanto para Solanum pimpinellifolium como para S. habrochaites, en condiciones de laboratorio, y que puedan ser exportadas a campo de cultivo.

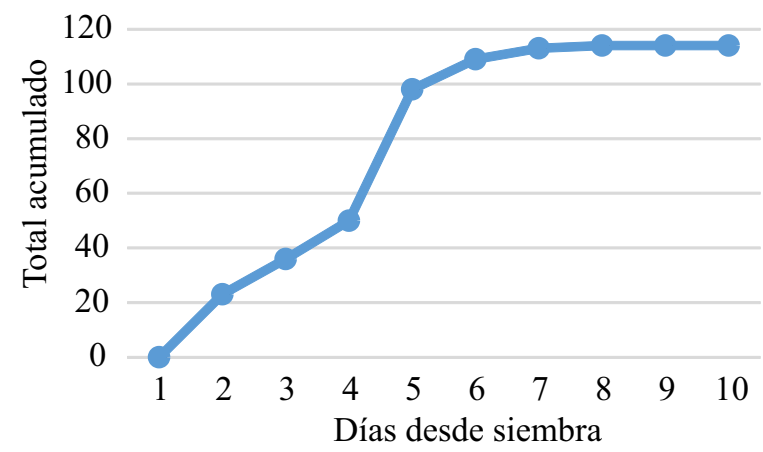

Figura 2. Total acumulado, en días de semillas germinadas, de Solanum pimpinellifolium en condiciones de laboratorio.

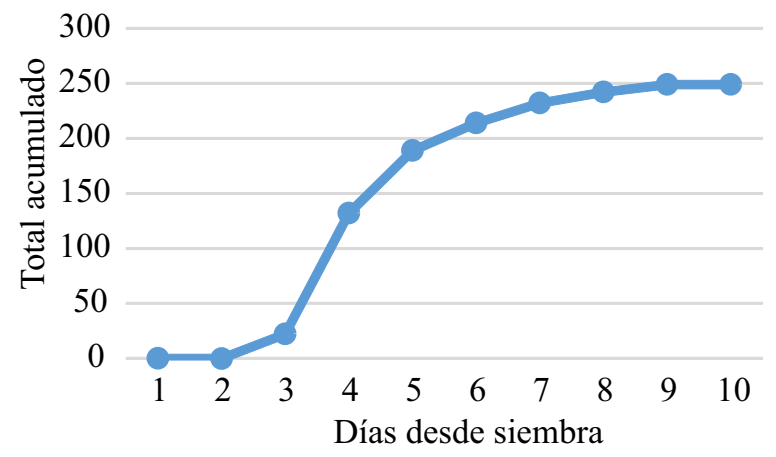

Figura 3. Total acumulado en días de semillas germinadas de Solanum habrochaites, en condiciones de laboratorio.

\section{IV.DISCUSIÓN}

El porcentaje de germinación del $76 \%$ reportado para Solanum pimpinellifolium, nos muestra el alto grado de adaptabilidad que tiene esta especie a ecosistema xerofíticos, como los de la loma del "Cerro Campana", y que concuerda con lo descrito por Tomalá (2015), quien obtiene mediante ensayos valores de porcentaje de germinación de entre el 60 y el 90 \%. Es decir el rango dado por este autor abarca el valor encontrado en nuestra investigación.

En cuanto al valor de "Velocidad de germinación" mostrado en este estudio, que alcanza los 4,87 días promedio, nos dice del gran potencial que tiene esta especie para colonizar cualquier hábitat por adversos que sea. Por ello que a partir del segundo día empezó el crecimiento logarítmico del proceso de germinación, valor que se estabilizó a los 10 días. Esto sumado a la “Uniformidad de germinación”, calculada en los 6,33 días desde la siembra, y en el porcentaje de emergencia, de 55,33\%, hacen de esta especie UN recurso importante para utilizarla en ensayos de investigación para obtener variedades y contribuir en la conservación y restauración de su hábitat. Al mismo tiempo puede servir para cruzamientos genéticos en la obtención de nuevos híbridos cultivables, aspectos que concuerdan con lo descrito por Pérez (2010), quien afirma que Solanum pimpinellifolium es una especie silvestre muy relacionada con el "tomate cultivado", que se cruza fácilmente con este, constituyéndose en una especie clave para futuros trabajos de mejoramiento genético.

En lo concerniente al "Valor de germinación", estimado en 31,02, según el índice de Czabator, refuerza aún más el grado de adaptabilidad que tiene la especie, concordando con lo ya descrito por Mostacero et al., (2007), quienes aseveran que esta especie originaria de los Andes peruanos y ecuatorianos, se extiende muy cómodamente desde la costa del Perú hasta el nororiente de Ecuador, con capacidad de colonizar cada uno de estos ecosistemas con mucha facilidad.

Paralelamente a lo hallado para Solanum pimpinellifolium, hasta el día de hoy no se han reportado datos sobre las características germinativas de la otra especie lomal, Solanum habrochaites, como especie promisoria en el mejoramiento genético de los "tomates comerciales" (Solanum lycopersicum). En cambio, los hallazgos de esta investigación demuestran que $S$. habrochaites también posee un elevado "Porcentaje de germinación", del 83 \%, una "Velocidad de germinación” de 5,6 días, tardando tan sólo un día en iniciar su germinación, y donde se aprecia el incremento logarítmico del proceso de germinación a partir del día 3, para luego estabilizarse a los 10 días. Este proceder es muy similar al de Solanum pimpinellifolium y Solanum lycopersicum, especies capaces de colonizar distintos ambientes, algo que sin duda han logrado, tras muchos años de selección natural y domesticación. 
Los resultados mencionados en el párrafo anterior sumados a la "Uniformidad de germinación" de 6,67 días, "Porcentaje de emergencia" de 49,33 \%, y al "Valor de la germinación" de 116,17, permiten afirmar que es estas especies son vitales tanto para mejorar la agroindustria de las mismas como para implementar programas de conservación y utilización racional de estas especies, ya que se trata de recursos no sólo resistentes a plagas de insectos y de hongos claves, sino también tolerantes a condiciones ambientales adversas, tal como lo afirman Paterson et al. (1987), que han encontrado a estas especies y a otras similares como tolerantes a heladas, y ricas en contenido en azúcares y ácidos orgánicos (Roselló y Nuez, 2006), justificando el hecho de ser calificadas como promisorias para el Perú e importantes para estos ecosistemas de las lomas del "Cerro Campana".

\section{CONCLUSIONES}

Los valores del "Porcentaje de germinación" del $76 \%$, una "Velocidad de germinación" de 4,87 días, una "Uniformidad de germinación" de 6,33 días, un "Porcentaje de emergencia" de 55,33 \%, y un "Valor de la germinación" de 31,02 , hacen que Solanum pimpinellifolium sea una especie promisoria tanto para el mejoramiento genético de especies cultivadas como para programas de conservación y sostén de las lomas como ecosistemas.

Igualmente el "Porcentaje de germinación" del $83 \%$, una "Velocidad de germinación" de 5,6 días, una "Uniformidad de germinación" de 6,67 días, un "Porcentaje de emergencia" de 49,33\%, y un "Valor de la germinación" de 116,17, hacen de Solanum habrochaites, una especie mejor que la anterior en relación a las perspectivas y tendencias mostradas, y lo catalogan como especie de mucho mejor valor para los fines comerciales.

\section{REFERENCIAS BIBLIOGRÁFICAS}

Bai, Y., y P. Lindhout. 2007. "Domestication and Breeding of Tomatoes: What have We Gained and What Can We Gain in the Future?" Annals of Botany 100: 1085-1094.

Bucheli, P. 1999. "Definition of biochemical and molecular markers (quality trait loci) for tomato flavour as tools in breeding". Acta horticulturae 1:301-306.

Canziani, J. 1998 “Las lomas de Atiquipa: un caso de paisaje natural en la costa desértica del sur del Perú". En Paisajes culturales en los Andes. Lima (Perú): CONDESAN.

Carranza, L. 1996. "Lomas del Cerro Campana": Estudio geológico y geomorfológico". Arnaldoa 1: 95-101.

Cortada, L. 2009. "Response of tomato rootstocks carrying the Mi-resistance gene to populations of Meloidogyne arenaria, M. incognita and M. javanica". European Journal of Plant Pathology 124: 337-343.

Dimock, M. 1983. "The role of glandular trichomes in the resistance of Lycopersicon hirsutum $f$. glabratum to Heliothis zea". Entomologia experimentalis et applicata 33: 263-268.

Ferreyra, R. 1979. Sinopsis de la Flora Peruana Gimnospermas y Monocotiledóneas. Lima (Perú): UNMSM.

Guo, Z., P. Weston, y J. Snyder. 1993. "Repellency to 2-spotted spider-mite, tetranychus-urticae koch, as related to leaf surface-chemistry of Lycopersicon hirsutum accessions". Journal of Chemical Ecology 19: 2965-2979.

Harman, A., y J. Bale. 1994. Propagación de plantas, principios y plántulas. Mexico D. F. (México): Editorial Continental.

Juvik, J. 1982. "Resistance among accessions of the genera Lycopersicon and Solanum to four of the main insect pests of tomato in Israel". Phytoparasitica 10: 145-156.

Kalloo, G. 1993. “Tomato”. En Genetic improvement of vegetable crops. Kalloo, G., y B.O. Bergh (eds). Londres (Reino Unido): Pergamon Press.

Kenedy, G. y R. Yamamoto. 1979. "Resistance in a wild tomato to the tobacco hornworm: the 
presence of a toxic factor". Entomologia experimentalis et aplicata 26: 121-126.

Lamb, D. 2011. Regreening the bare hills: Tropical forest reforestation in the Asia Pacific region. New York (EEUU): Springer.

Leite, G., M. Picanco, R. Guedes, y J. Zanuncio. 2001. "Role of plant age in the resistance of Lycopersicon hirsutum f. glabratum to the tomato leafminer Tuta absoluta (Lepidoptera: Gelechiidae)". Scientia horticulturae 89: 103-113.

Lin, S. 1987. "Activity of volatile compounds in glandular trichomes of Lycopersicon species against two insect herbivores". Journal of chemical ecology 13: 837-850.

Morales, G., y M. Camacho. 1985. "Formato y recomendaciones para evaluar germinación". En III Reunión Nacional sobre plantaciones Forestales. México D.F. (México).

Mostacero, J. 1987. Aspectos Fitogeográfico de las Lomas de la provincia de Trujillo (La Libertad). Trabajo de habilitación para ascenso de Categoría docente. Universidad Nacional de Trujillo. Trujillo (Perú).

Mostacero, J., F. Mejía, W. Zelada, y A. Medina. 2007. Biogeografía del Perú. Trujillo (Perú): UNT.

Paterson, B., L. Mutton, R. Paull, y V. Nguyen. 1987. "Tomato pollen development: stages sensitive to chilling and a natural environment for the selection of resistant genotypes". Plant Cell and Environment 10:363-368.

Pérez, M. 2010. Mejoramiento genético en Solanum lycopersicum para la resistencia al pasador del fruto Neoleucinodes elegantalis Guenée (Lepidoptera: Crambidae). Tesis de Maestría. Universidad Nacional de Colombia. Bogotá (Colombia)

Quiroz, M. 2008. La Montaña Sagrada Campana. Trujillo (Perú): UPAO.

Rick, C. 1991 "Tomato paste: a concentrated review of genetic highlights from the beginnings to the advent of molecular genetics". Genetics 128:
$1-5$.

Rick, C., J. Fobes, y S. Tanksley. 1979. "Evolution of mating systems in Lycopersicon hirsutum as deduced from genetic variation in electrophoretic and morphological characters". Plant Systematics and Evolution 132: 279298.

Roselló, S., y F. Nuez. 2006. "Mejora de la calidad del tomate para fresco". En Mejora genética de la calidad en plantas. Llácer, G., M. J. Díez, J. M. Carrillo, M. L. Badene (eds). Buenos Aires (Argentina).

Sagástegui, A., J. Mostacero, y S. López. 1988. "Fitoecología del Cerro Campana". Bol. Soc. Bot. de la Libertad 14: 1-47.

Tomalá, J. 2015. Capacidad germinativa del genotipo de tomate riñón (Lycopersicon esculentum Mill.) al estrés salino en diferentes fotoperiodos. Tesis de Grado. Universidad Estatal Península de Santa Elena. La Libertad (Ecuador.

Webb, R., A. Stoner, y A. Gentile. 1971. "Resistance to leafminers in Lycopersicon accessions". Journal of the American Society for Horticultural Science 95: 65-67.

Williams, E., G. Kenedy, R. Yamamoto, J. Thacker, y J. Bordner. 1980. "2- tridecadone: a naturally occurring insecticide from the wild tomato Lycopersicon hirsutum f. glabratum". Science 207: 880-888.

Zuriaga, E., J. Blanca, L. Cordero, y A. Sifres. 2009. "Genetic and bioclimatic variation in Solanum pimpinellifolium". Genet Resour Crop Evol 56: 39-51. 